Jack TANNous, The Making of Medieval Middle East: Religion, Society, and Simple Believers

\title{
Antony Roch
}

\section{(2) OpenEdition}

\section{Journals}

Édition électronique

URL : https://journals.openedition.org/ccm/5108

DOI : $10.4000 / \mathrm{ccm} .5108$

ISSN : 2119-1026

\section{Éditeur}

Centre d'études supérieures de civilisation médiévale/Université de Poitiers

\section{Édition imprimée}

Date de publication : 1 septembre 2020

Pagination : 194-196

ISBN : 978-2-490783-06-9

ISSN : 0007-9731

\section{Référence électronique}

Antony Roch, " Jack TAnnous, The Making of Medieval Middle East: Religion, Society, and Simple Believers », Cahiers de civilisation médiévale [En ligne], 250-251 | 2020, mis en ligne le 01 septembre 2020, consulté le 03 décembre 2022. URL : http://journals.openedition.org/ccm/5108 ; DOI : https:// doi.org/10.4000/ccm.5108

\section{cc) (i) (9)}

Creative Commons - Attribution - Pas d'Utilisation Commerciale - Pas de Modification 4.0 International - CC BY-NC-ND 4.0

https://creativecommons.org/licenses/by-nc-nd/4.0/ 
Jack Tannous, The Making of Medieval Middle East: Religion, Society, and Simple Believers, Princeton/ Oxford, Princeton University Press, 2018.

L’historien américain Jack Tannous nous livre ici une étude de grande érudition et originale à plus d'un titre. Cet ouvrage entend analyser en profondeur la situation sociale, théologique et de la vie quotidienne des chrétiens du Moyen-Orient dans les premiers siècles de la domination musulmane. En somme, le monde que les arabo-musulmans découvrirent lorsqu'ils conquirent la région. La première originalité de l'ouvrage est avant tout la focalisation sur les populations chrétiennes de la région, quand d'autres études se sont concentrées avant tout sur la conquête musulmane et les populations arabes arrivantes. Il rappelle de ce fait une situation bien souvent oubliée et parfois niée : la population chrétienne fut largement majoritaire dans la région conquise, et cela quasiment jusqu'à l'époque des croisades. Le livre se découpe en quatre parties, elles-mêmes divisées en chapitres (14 au total). La structuration suit un canevas cohérent permettant de saisir avec clarté la démonstration de l'a. En outre, J. Tannous appuie son argumentation par de nombreux extraits de sources premières variées, et très riches d'information.

Les deux premières parties de l'ouvrage sont consacrées à la construction du cadre socio-théologique que les conquérants arabes ont trouvé lors de l'annexion du Moyen-Orient. La première partie entend traiter la question des croyances de base des populations. Avant tout, il s'agit pour l'a. de traiter une double perspective. Premièrement, J. Tannous observe celle entre la spéculation théologique et ce qu'il nomme alphabétisation théologique («theological literacy»), dont l'application concerne principalement la compréhension des concepts de définition christologique développés par les premiers conciles, et acceptés ou rejetés par certaines populations. L'a. estime difficile d'apporter un pourcentage précis au degré d'alphabétisation du Moyen-Orient vers le vIII ${ }^{\mathrm{e}} \mathrm{s}$., mais avance tout de même les chiffres de 5 à $10 \%$ de personnes alphabétisées. Le bas niveau de connaissances littéraires, couplé au manque d'accès aux livres, est ainsi bien mis en lumière. Deuxièmement, la relation entre simples croyants et croyants érudits dans la période post-Chalcédoine est mise en avant, les élites étant quasiment notre seul accès à une description des simples croyants. Les sources étudiées dans ce chapitre nous renseignent sur les différences de conceptions théologiques potentielles entre ces dissemblables catégories de populations, les communautés chrétiennes du Moyen-Orient étant majoritairement agrariennes et illettrées, souvent marquées par un rejet des conceptions théologiques complexes de l'élite lettrée. Il met ainsi en évidence la fragmentation des populations chrétiennes orientales face aux tentatives d'affirmation et de normalisation des conceptions du concile de Chalcédoine (451).

La deuxième partie de l'ouvrage, subdivisée en cinq chapitres, étudie directement les conséquences produites par le concile de Chalcédoine. L'a. observe en premier lieu l'état de confusion existant à la fin du $\mathrm{VII}^{\mathrm{e}} \mathrm{S}$., due à la coexistence (rivale comme amicale) entre les différents courants christologiques. Il relève la difficulté réelle que les définitions chalcédoniennes eurent pour tenter de s'imposer. J. Tannous relève à travers les sources la fréquentation par des populations d'une certaine confession chrétienne dans des églises d'un courant concurrent, ceci jusqu' au IX ${ }^{\mathrm{e}} \mathrm{s}$., soulevant l'ire des membres des clergés respectifs. L'a. émet l'hypothèse que la confusion doctrinale soit notamment due à la grande diversité socio-ethnique du Proche et Moyen-Orient. Les populations non lettrées ne faisaient néanmoins aucune distinction réelle entre les différents courants doctrinaux, créant une profonde confusion face aux élites lettrées qui tentaient de leur imposer ces distinctions. Cette situation conduisait inévitablement à des conflits, parfois violents, mais avant tout intellectuels, à travers 
des débats, des disputes et la narration d'histoires. Deux individus de deux courants différents pouvaient souvent s'entraider dans leur questionnement théologique. Mais l'utilisation d'histoire de propagande narrant la victoire fantastique d'une confession sur une autre met en exergue les outils que les chefs d'Églises avaient à disposition dans un contexte social qui ne permettait pas une formation théologique adéquate, ni un accès aux livres nécessaires pour comprendre les problèmes soulevés par Chalcédoine et ses portées doctrinales. J. Tannous observe en outre que l'utilisation des sacrements a joué un rôle prépondérant dans les luttes d'influences entre divers courants théologiques, et s'est même étendu aux groupes non chrétiens. Ces luttes entraînèrent les différents courants à créer des institutions d'éducation pour tenter de conserver leurs ouailles ou en gagner de nouvelles. L'a. entend de ce fait également étudier l'articulation de l'éducation chrétienne dans le Moyen-Orient post-chalcédonien. Il conclut cette deuxième partie en faisant le constat d'une société moyen-orientale peuplée de simples croyants, appartenant à des Églises diverses, tiraillés par des élites dirigeantes religieuses promptes à ériger des barrières entre elles, et à fracturer ainsi le paysage confessionnel de la région. C'est ce paysage socio-confessionnel que les Arabes découvrirent au VII ${ }^{\mathrm{e}} \mathrm{s}$.

Dans un court interlude entre la deuxième et la troisième partie de son ouvrage, l'a. pose une interrogation essentielle, à savoir quelle influence allait avoir l'arrivée comme force politique dominante, le principal rival et critique théologique de la Chrétienté, notamment en rapport des dogmes trinitaires et de l'Incarnation, que les simples croyants n'étaient pas à même de comprendre pleinement.

Dans la troisième partie de son ouvrage, J. Tannous entend décrire les rapports socio-religieux entre les chrétiens et musulmans, relations entre croyants et dynamiques de conversions. Si à terme, la conquête arabo-musulmane conduisit à l'islamisation et arabisation de tout le Moyen-Orient, l'a. nous invite à définir la nature de cette conversion, et de quel christianisme et quel islam parlons-nous pour la deuxième moitié $\mathrm{du} \mathrm{VII}^{\mathrm{e}} \mathrm{s}$. Il démontre ainsi, à travers la présentation de sources riches d'informations, que le christianisme que ces nouveaux convertis - issus généralement de populations non lettrées, ces fameux simples croyants - ne correspondait en rien au christianisme doctrinal des élites, et que l'islam qu'il rejoignait ne correspondait nullement à l'islam sophistiqué de la Bagdad du IX $X^{\mathrm{e}}$ s., ou de l'islam politico-culturel du califat Omeyyade. J. Tannous, après avoir démontré le large éventail de signification que le mot « chrétien » pouvait impliquer, applique une méthode similaire afin de définir ce que signifiaient les mots « islam » et « musulman » pour la période concernée. L'a. décrit alors une situation aussi multiple pour l'islam fraîchement né que pour le christianisme moyen-oriental. Il y avait donc une multitude de compréhensions directes de la religion musulmane parmi les arrivants et les nouveaux convertis. Autre point important, il apparaît que la conversion collective, ou clanique, eut été la règle, et que les conversions individuelles basées sur des considérations théologiques liées au Coran et à la figure prophétique de Muhammad ne furent que l'exception. Le phénomène de conversion de masse de certains groupes de populations arabes qui arrivèrent avec la conquête laisse entrevoir une majorité de musulmans purement et simplement ignorants de toute connaissance théologique. Les sources laissent apparaître que les élites instruites elles-mêmes s'inséraient dans une diversité de croyances qui s'entendaient comme légitimes, comprenant des attitudes tout aussi diverses par rapport au Coran lui-même. Cette diversité doit être prise ainsi en compte lorsqu'on cherche à définir les rapports entre chrétiens et musulmans à cette période.

J. Tannous rappelle une donnée particulièrement importante : les musulmans n'étaient alors qu'une petite minorité dans les années de conquête du MoyenOrient. Il révèle que le phénomène de conversion ne se révélait pas toujours définitif, et qu'il fut dans l'ensemble, un processus relativement long. La majorité des musulmans descendaient alors de convertis autrefois non musulmans, de sorte que la construction de ce qui deviendra l'islam normatif se fit au travers d'un ensemble de réponses au problème des populations non musulmanes. Les simples chrétiens étudiés dans la première partie sont devenus progressivement de simples musulmans, peu éduqués sur leurs traditions religieuses et globalement mal équipés pour le débat théologique complexe. L'a. nous invite également à dépasser l'enjeu des seules différences théologiques, afin de considérer les processus d'échanges interconfessionnels qui se sont manifestés. Il révèle ainsi que les premières générations musulmanes vivant sur ces territoires conquis ont eu recours, pour certaines d'entre elles, au rite du baptême et utilisèrent également le symbole de la croix, malgré d'autres comportements plus hostiles à ces rites et symboles. Il relève également la profonde influence que l'architecture des églises a eue sur celle des mosquées. Il faut également considérer les processus de contact et de transmission à travers les différents milieux où ceux-ci ont pu s'établir. J. Tannous révèle ainsi une réalité quotidienne où les populations chrétiennes 
et musulmanes se rencontraient dans les villes de garnisons, les monastères et les festivités, tous comme dans les maisons elles-mêmes. Il relève également que c'est à travers les convertis que les conquérants arabes et l'islam absorbèrent les pratiques et idées de l'Antiquité tardive.

En guise de conclusion, 1'a. invite une fois encore à reconsidérer les idées reçues sur la réalité démographique du Moyen-Orient médiéval. La perspective historiographique qu'il porte sur le sujet s'avère tout à fait novatrice. Concernant les communautés chrétiennes, il invite à ne pas les considérer exclusivement à travers des critères théologiques, les croyants - et une partie du clergé - ayant été en grande majorité purement et simplement indifférents, et bien souvent ignorants, à ce sujet. Il récuse par la même occasion la tendance à ne saisir l'histoire du MoyenOrient des $\mathrm{VII}^{\mathrm{e}}$ et $\mathrm{VIII}^{\mathrm{e}} \mathrm{s}$. en la reliant qu'à l'histoire islamique, alors que la population fut en majeure partie chrétienne pendant près d'un demi-millénaire suivant la conquête. Il considère au contraire au centre de son ouvrage cette part majoritaire des non musulmans, en particulier les chrétiens, et met de sorte en évidence les liens de continuités religieuses et culturelles malgré les ruptures occasionnées par la conquête arabe.

J. Tannous invite pour finir à un appendice précieux consacré à l'approche des sources utilisées. Celui-ci s'avère éclairant sur la méthodologie employée par l'a. pour développer son argument. Apparaît notamment le problème majeur, celui de devoir utiliser des sources d'auteurs issus des élites lettrées pour aborder les simples croyants, alors qu'il cherchait précisément à éviter ces élites. Sa tendance est également de naviguer entre des sources très différentes de natures et d'époques. Néanmoins, son utilisation des sources prend sens dans l'argumentation générale. Un dernier appendice entend poser une réflexion sur le terme syriaque tayyāye , utilisé pour décrire les conquérants arabes. L'ouvrage de 647 p. s'achève finalement par une bibliographie dont il faut relever la grande richesse, et qui constitue de fait une base de données essentielle pour tout chercheur désireux de poursuivre l'étude du paysage socio- et politico-religieux du Moyen-Orient au haut Moyen Âge. Elle est suivie par un index général tout à fait utile.

Dans le Moyen-Orient actuel ravagé par des décennies de conflits, secoué par des mouvements de populations fuyant les zones de guerre, et où les chrétiens d'Orient ne sont plus qu'une petite minorité, dont la survivance apparaît comme précaire, nous avons tendance à oublier que cette situation ne fut pas immuable. Le présent ouvrage nous rappelle avec une précision inégalée jusqu'alors que cette transformation ne s'effectua que lentement, à partir de la conquête démarrée au VII ${ }^{\mathrm{e}} \mathrm{s}$. L'ouvrage de J. Tannous apparaît dès lors comme un remède puissant à l'oubli, et constitue de fait une somme incontournable dans l'étude du processus d'islamisation du Moyen-Orient, de transformations, de continuités et de ruptures religieuses et culturelles qui ont forgé le paysage socioculturel et politico-religieux de cette région, et nous invite à reconsidérer les stéréotypes parfois tenaces.

Antony RoCH Université de Fribourg $(\mathrm{CH})$ 